\title{
Estimation, Filtering and Fusion for Networked Systems with Network-Induced Phenomena: New Progress and Prospects
}

\author{
Jun $\mathrm{Hu}^{a, *}$, Zidong Wang ${ }^{b, c}$, Dongyan $\mathrm{Chen}^{a}$ and Fuad E. Alsaadi ${ }^{c}$
}

\begin{abstract}
In this paper, some recent advances on the estimation, filtering and fusion for networked systems are reviewed. Firstly, the network-induced phenomena under consideration are briefly recalled including missing/fading measurements, signal quantization, sensor saturations, communication delays, and randomly occurring incomplete information. Secondly, the developments of the estimation, filtering and fusion for networked systems from four aspects (linear networked systems, nonlinear networked systems, complex networks and sensor networks) are reviewed comprehensively. Subsequently, some recent results on the estimation, filtering and fusion for systems with the network-induced phenomena are reviewed in great detail. In particular, some latest results on the multiobjective filtering problems for time-varying nonlinear networked systems are summarized. Finally, conclusions are given and several possible research directions concerning the estimation, filtering, and fusion for networked systems are highlighted.
\end{abstract}

\section{Index Terms}

Estimation; filtering; multi-sensor data fusion; networked systems; network-induced phenomena.

\section{INTRODUCTION}

The networked systems have attracted increasing research attention due to their successful applications in a wide range of areas, such as aircraft, space and terrestrial exploration, access in hazardous environments, factory automation, remote diagnostics and troubleshooting, automated highway systems, unmanned aerial vehicles, manufacturing plant monitoring and condition-based maintenance of complex machinery [1]. The

This work was supported in part by the National Natural Science Foundation of China under Grants 61329301, 61273156, 11301118 and 11271103, the Youth Science Foundation of Heilongjiang Province of China under Grant QC2015085, the China Postdoctoral Science Foundation under Grants 2015T80482 and 2014M560376, Jiangsu Planned Projects for Postdoctoral Research Funds under Grant 1402004A, Science Funds for the Young Innovative Talents of HUST, the Royal Society of the U.K., and the Alexander von Humboldt Foundation of Germany.

a Department of Applied Mathematics, Harbin University of Science and Technology, Harbin 150080, China. (Email: hujun2013@gmail.com)

${ }^{b}$ Department of Computer Science, Brunel University London, Uxbridge, Middlesex, UB8 3PH, United Kingdom. (Email: Zidong.Wang@brunel.ac.uk)

${ }^{c}$ Communication Systems and Networks (CSN) Research Group, Faculty of Engineering, King Abdulaziz University, Jeddah 21589, Saudi Arabia.

* Corresponding author. E-mail: hujun2013@gmail.com 
advantages of the usage of networked systems include flexible architecture, the reduction of installation and maintenance costs, decreasing the implementation difficulties and so on. However, the network-induced phenomena arise inevitably due to the insertion of the communication network with limited communication capacity [2]-[5]. Such network-induced phenomena include, but are not limited to, communication delays, missing/fading measurements, signal quantization, sensor saturations, variable sampling/transmission intervals, and out-of-sequence-measurement updates. Recently, a class of newly emerged network-induced phenomena (randomly occurring incomplete information) has gained some initial research interest in signal processing and control areas. Note that the network-induced phenomena could greatly degrade the performance of the networked systems and may even lead to the instability of the controlled systems [6], [7]. Consequently, it is not surprising that both analysis and synthesis problems for networked systems have received considerable research attention in the past decade.

The filtering problem has long been one of the foundational research problems in signal processing and control engineering [8]-[12]. The past two decades have witnessed the rapid developments and extensive applications of the filtering algorithms in practice, such as guidance, navigation, target tracking, remote sensing, image processing, econometrics, and monitoring of manufacturing processes. Therefore, the design of the filtering algorithms has received increasing research attention. According to different performance indices (minimized variance constraint, set-valued constraints, guaranteed $H_{\infty}$ performance requirements and so on), a great number of filtering algorithms have been developed for networked systems, such as Kalman filtering [13], [14], extended Kalman filtering [15]-[18], set-valued filtering [19], [20], setmembership filtering [21], $H_{2}$ filtering [22]-[24], $H_{\infty}$ filtering [25], [26], and consensus filtering [27], [28]. On the other hand, the design of linear optimal estimators (including filter, predictor and smoother) for networked systems has gained a great deal of research attention as conducted in [29]-[32].

On another research frontier, it is well known that the data fusion techniques can provide the fusion schemes by combining the information from different sources so as to achieve a satisfactory performance. Over the past decades, the data fusion techniques have been successfully applied in a variety of areas such as the target tracking, navigation, detection, robotics, video and image processing, business intelligence, and sensor networks. Therefore, considerable research effort has been devoted to the multi-sensor data fusion problems for complex dynamical systems. In fact, as mentioned in [33], there are a great number of challenging issues in the multi-sensor data fusion fields including data imperfection, outliers and spurious data, conflicting data, data modality, data correlation, data association, data alignment/registration, processing framework, operational timing, static versus dynamic phenomena, data dimensionality and so on. For more information about the challenging problems of the multi-sensor data fusion, we refer the readers to the survey paper [33] where more comprehensive interpretations have been provided. In what follows, we confine the addressed topic to the multi-sensor data fusion for networked systems and endeavor to introduce some recent advances on the network-based multi-sensor data fusion approaches from the perspective of algorithm developments. The multi-sensor data fusion algorithms can be generally classified into two types: centralized fusion and distributed fusion algorithms, where the schematic diagrams of centralized and distributed fusions in network environment are given as in Figs. 1-2 respectively. We will 
further discuss the recent developments of the multi-sensor fusion of networked systems later.

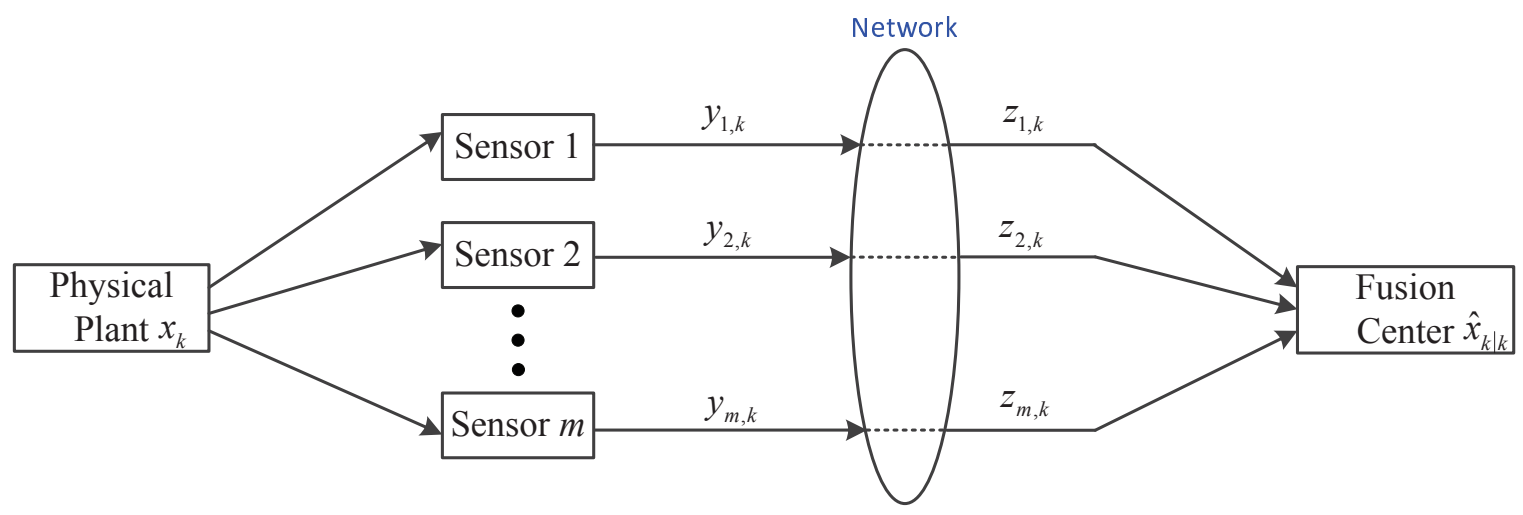

Fig. 1. Schematic structure of centralized fusion over network

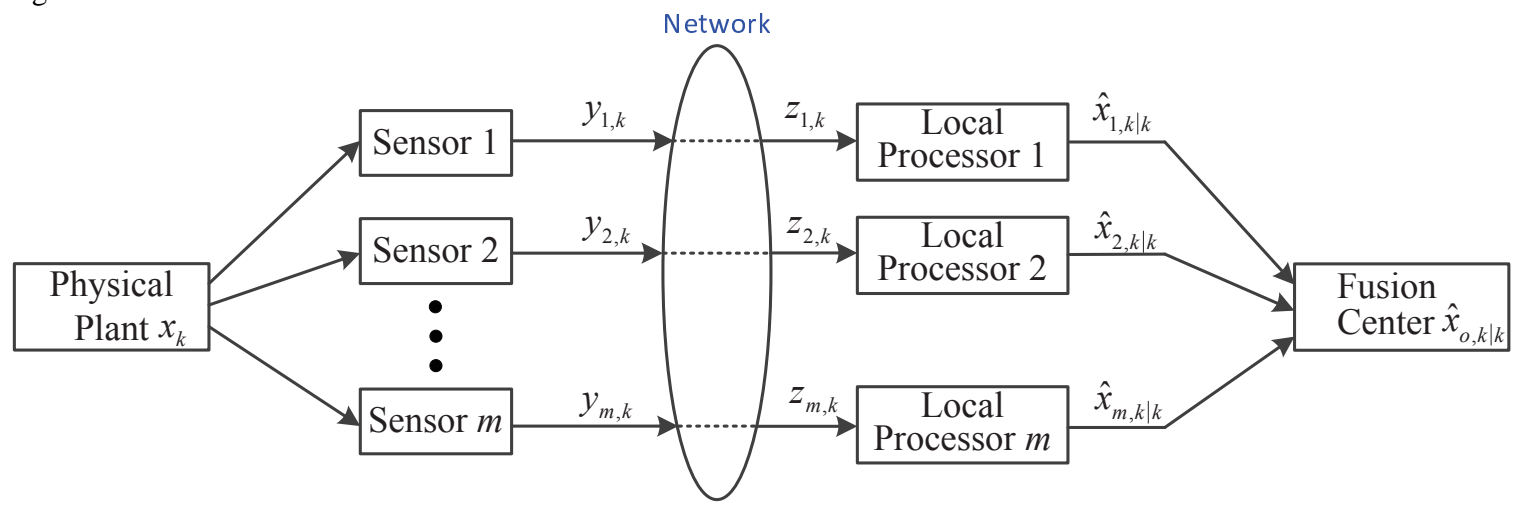

Fig. 2. Schematic structure of distributed fusion over network

In this paper, we aim to provide a timely review on the recent advances of the estimation, filtering and fusion algorithms for networked systems with network-induced phenomena. The addressed networkinduced phenomena include missing/fading measurements, communication delays, signal quantization, sensor saturations, randomly occurring uncertainties, randomly occurring nonlinearities, randomly occurring signal quantization, randomly occurring sensor saturations and so on. The recent developments of the network-induced phenomena are firstly discussed. Secondly, we review the analysis and synthesis problems of the networked systems from four aspects, including linear networked systems, nonlinear networked systems, complex networks and sensor networks. In the same section, several estimation, filtering and fusion schemes for networked systems are surveyed in great detail. Thirdly, latest results on estimation, filtering and fusion approaches for networked systems with network-induced phenomena are reviewed. Finally, conclusions are drawn and some possible research directions are pointed out.

The remainder of this paper is organized as follows. In Section II, the network-induced phenomena are discussed. In section III, the developments of the estimation, filtering, fusion problems for networked systems are summarized. In section IV, some latest results on the estimation, filtering and fusion problems for complex dynamical systems with network-induced phenomena are reviewed. Both conclusions and some future research topics are provided in section $\mathrm{V}$. 


\section{Network-INDUCED PHENOMENA}

Over the past decade, a great deal of research attention has been received regarding the modeling and analysis of the network-induced phenomena including missing/fading measurements, signal quantization, sensor saturations, communication delays, variable sampling/transmission intervals, out-of-sequencemeasurement updates, randomly occurring incomplete information etc. Accordingly, many important approaches have been given to examine the effects from the network-induced phenomena onto the system performance. In this section, some representative network-induced phenomena will be briefly reviewed.

\section{A. Missing/Fading Measurements}

The traditional estimation and filtering algorithms rely on an ideal assumption that the measurement outputs are available always. Nevertheless, the imperfect communication would occur in practical engineering especially in the networked systems, namely, the measurement outputs may contain noise only at certain instants and the desired signals are missing due probably to temporal sensor failures or network transmission delay/loss [34]-[39]. During the past two decades, among the probabilistic ways for modeling the missing measurements, the Bernoulli probability distribution has been extensively employed due to its simplicity and practicality, where the Bernoulli random variable takes value on 1 representing the perfect signal delivery and it takes value on 0 standing for the measurement missing. Accordingly, many important papers have been published concerning on the estimation, filtering and fusion for networked systems based on several methods such as the linear matrix inequality method [25], difference linear matrix inequality method [27], innovation analysis approach [30], Hamilton-Jacobi-Isaacs inequality approach [35], and backward/forward Riccati difference equation method [7], [37]. When comparing between different approaches, it is worth mentioning that the linear matrix inequality (difference linear matrix inequality) method is applicable for the analysis problem of time-invariant (time-varying) linear/nonlinear networked complex dynamical systems and gives the feasible solutions, the innovation analysis approach is suitable for handling the analysis problem of linear time-invariant/time-varying networked systems and can provide the optimal solutions in the minimum mean-square error sense, the Hamilton-JacobiIsaacs inequality approach is helpful for addressing the analysis and synthesis problems of time-invariant networked systems with general nonlinearities but it is commonly difficult to obtain the feasible solution, and backward/forward Riccati difference equation method has the advantage to deal with the analysis and synthesis problem for time-varying linear/nonlinear networked systems and provide the sub-optimal solutions. A more detailed comparison is given in Table I with hope to better understand the differences among the existing methods.

On the other hand, the measurement signals during the network transmissions may fade/degrade in a probabilistic way rather than be lost completely [49], [51], [52], [55]-[57]. It is easy to see that the missing measurements are extreme cases of the fading measurements. By using sequences of random variables obeying a certain probability distribution over known intervals with available conditional probabilities, the phenomena of the multiple fading measurements have been modeled in [52] and a Kalman-like recursive filtering algorithm has been developed via the forward Riccati difference equation approach. Besides, in 
TABLE I

COMPARISONS AMONG DIFFERENT METHODS

\begin{tabular}{|c|c|c|c|}
\hline Methods & Applications & Solutions & References \\
\hline Linear matrix inequality method & time-invariant complex dynamical systems & feasible & {$[25],[40]-[44]$} \\
\hline Difference linear matrix inequality method & time-varying complex dynamical systems & feasible & [21], [27], [45], [46] \\
\hline Innovation analysis approach & linear time-invariant/time-varying systems & optimal & {$[30]-[32],[39],[47],[48]$} \\
\hline Hamilton-Jacobi-Isaacs inequality approach & general nonlinear time-invariant systems & feasible & [35] \\
\hline Backward Riccati difference equation method & nonlinear time-varying systems & sub-optimal & [37], [49], [50] \\
\hline Forward Riccati difference equation method & nonlinear time-varying systems & sub-optimal & {$[7],[16],[51]-[54]$} \\
\hline
\end{tabular}

[49], [56], [57], the $N$-order Rice fading channel has been modeled by sequences of independent and identically distributed Gaussian random variables with known means and variances, where the multi-path induced fading stemming mainly from multi-path propagation has been considered when dealing with the control and estimation problems for networked systems and the impact from the fading measurements onto the control/estimation performance has been examined.

\section{B. Signal Quantization}

In the networked environment, signals are often quantized before the transmissions because of the finite-word length of the packets [58]-[61]. During the implementation, a device or algorithmic function performing the quantization is called a quantizer and an analog-to-digital converter can be seen as an example of a quantizer. Note that the signal quantization would affect the achievable performance of the networked systems and, hence, there is a need to conduct the analysis on various quantizers and examine the effects from the quantization onto the system performance. Recently, the signal quantization problem has become a research focus and attracted an ever-increasing interest. Accordingly, some methods have been proposed in [62]-[64] to handle the uniform quantization (the quantizers have same sensitivity) and in [65]-[67] to deal with the logarithmic quantization (the quantization levels are linear in logarithmic scale). As discussed in [68], a logarithmic quantizer can provide better efficiency in terms of the data rate for system performance than a uniform quantizer. So far, a great deal of effort has been devoted to address the filtering problems for networked systems with signal quantization and some effective filtering algorithms have been developed in [53] with variance constraints and in [69], [70] with $H_{\infty}$ performance requirements. For example, the fault detection filtering algorithms have been given in [69], [70] for linear networked systems with logarithmic quantization by using the linear matrix inequality technique. However, it is worthwhile to mention that most reported results have been concerned with time-invariant networked systems with signal quantization only and the corresponding filter design problem for timevarying networked systems has not been paid adequate research attention.

\section{Sensor Saturations}

As is well known, sensors may not always be capable of providing signals with unlimited amplitudes due to physical/technological restrictions. The occurrence of the sensor saturations could affect the imple- 
mentation precision of the developed filtering algorithms and may even cause severe degradation of the filtering performance if not handled properly. In the past ten years, the filtering problems for networked systems with sensor saturations have gained some initial research attention and some preliminary results have appeared handling the sensor saturations in recent literature [71], [72]. The main challenge with this topic is how to design a filtering algorithm by making full use of the available information about the sensor saturations subject to specified performance requirements (minimized variance, guaranteed $H_{\infty}$ constraints etc). Recently, by using the sector-bounded approach in [73], [74], a decomposition technique has been given to facilitate the filter design for networked systems and a great number of papers have been published. For example, in [75], a probability-guaranteed $H_{\infty}$ performance index has been defined over a finite-horizon, and a probability-guaranteed $H_{\infty}$ filtering algorithm has been developed for a class of time-varying nonlinear networked systems subject to random parameter matrices and sensor saturations. However, when it comes to the variance-constrained filtering and estimation problems for time-varying nonlinear networked systems with sensor saturations, the related results are very few and the situation is even worse when the randomly occurring incomplete information is also considered.

\section{Communication Delays}

The communication delays are frequently encountered in modern industrial systems (chemical process, long transmission lines in pneumatic, and communication networks) due to the finite switching speed of amplifiers or finite speed of information processing [76]-[84]. In the past two decades, many efficient approaches have been given to reduce the conservatism caused by the time delays, such as the bounding technique [85], the descriptor system method [86], the slack matrix variables technique [87] and the delayfractioning approach [88], [89]. Generally speaking, the objective of conducting the delay-dependent analysis includes two aspects (conservatism and complexity): 1) development of the delay-dependent conditions to provide a maximal allowable delay; and 2) development of the delay-dependent conditions by using as few decision variables as possible while achieving the same maximal allowable delay. When comparing between different methods, both the conservatism and the complexity serve as the criteria, and there exists a tradeoff between the conservatism and the complexity. Hence, it is difficult to look for a globally optimal approach which is least conservative yet with least computational burden. Compared with the bounding technique, the slack matrix variables technique and the descriptor system method, the delayfractioning approach is efficient in reducing the conservatism caused by the time-delays at the cost of introducing more computational complexity especially when the number of fractions goes up. Fortunately, it is not difficult to handle the computational complexity problem nowadays due to the rapid developments of the computing techniques. Based on the reported delay analysis methods, a great number of results have been published concerning the synthesis problem of the time-delay systems. Note that most of the relevant results have been concerned with the deterministic delays only, while the communication delays induced by network transmissions would be random and time-varying. As such, the random communication delays have received some initial research interests and the problems of estimation, filtering and fusion have been studied for networked systems with random communication delays [39], [90]-[93]. For example, 
the filtering problems have been studied in [39], [90], [92], [93] for networked systems with random communication delays modeled by Bernoulli random variables. In [91], the optimal filtering problem has been investigated for networked systems with random communication delays modeled by Markov chain.

\section{E. Randomly Occurring Incomplete Information}

Recently, accompanying with the increasing of the network scale, the randomly occurring incomplete information has become a hot research topic that has gained some initial research attention. The randomly occurring incomplete information may occur intermittently in a probabilistic way with certain types and intensity. For example, in a networked system such as the internet-based three-tank system for leakage fault diagnosis, the nonlinearities may occur in a probabilistic way due to random abrupt variations and the occurrence probability can be estimated via the statistical tests [94]. It is well recognized that the existence of the randomly occurring incomplete information would highly degrade the system performance if not handled properly. So far, a series of estimation and filtering schemes has been developed for networked systems with randomly occurring incomplete information in the literature, and great efforts have been made to deal with the randomly occurring nonlinearities in [49], [95]-[99], the randomly occurring uncertainties in [94], [97], the randomly occurring sensor saturations in [40], [72], the randomly occurring sensor delays in [31], [32], [38], [100], [101], the randomly occurring signal quantization in [41], [102], and the randomly occurring faults in [103]. Accordingly, several techniques for analysis and

synthesis of the networked systems have been given, including innovation analysis approach [31], [32], linear matrix inequality approach [97], Hamilton-Jacobi-Isaacs inequality method [100], difference linear matrix inequality method [41], Riccati difference equation approach [101], [102], and game theory method [54].

\section{ANALYSIS AND Synthesis OF NeTWORKED Systems}

Over the past two decades, the networked systems have been received an ever-increasing research attention due to its engineering insights in a variety of areas such as the guidance and navigation, air traffic control, factory automation, remote diagnostics and troubleshooting and automated highway systems [104]-[107]. In this section, the methodologies of modeling, estimation, filtering and fusion for networked systems in the literature are briefly surveyed.

\section{A. Linear Networked Systems}

During the past decade, the estimation problems of the linear networked systems have gained considerable research attention and a great number of methods have been given including innovation analysis approach, linear matrix inequality method, game theory approach, etc. For example, the linear optimal estimation problems have been studied in [30]-[32], [39] for linear discrete time-varying networked systems with packet dropouts, and the linear optimal estimators (including filter, predictor and smoother) have been designed based on the innovation analysis approach. Due to the limited capacity of the communication networks, the multiple network-induced phenomena (random transmission delays, packet 
dropouts) may occur simultaneously during the signal transmissions. For instance, in [31], [32], both the random transmission delays and the packet dropouts have been discussed in a unified framework. Compared with the results in [30], it is worth mentioning that the consecutive packet dropouts in [31] are finite and the consecutive packet dropouts in [32] can be infinite. In contrast to the modeling method of the random transmission delays based on the Bernoulli probability distribution in [31], [32], the phenomenon of random transmission delays has been modeled in [91] by a multi-state Markov chain and the optimal filtering problem has been studied for networked systems subject to random transmission delays. To further reflect the engineering reality and improve the estimation performance, the phenomena of random transmission delays and packet dropouts occurring in two sides (from sensor to estimator and from controller to actuator) have been modeled in [92] within a unified framework, and the optimal estimators (including filter, predictor and smoother) in the linear minimum variance sense have been designed by using the orthogonal projection approach.

When the state-space model of the signal is unknown, some estimation algorithms for linear networked systems can be found in the literature [47], [48]. To be specific, based on the innovation analysis approach, the linear recursive filtering and smoothing algorithms have been presented in [47] to handle the phenomenon of multiple random delayed measurements with different delay rates, and the recursive leastsquares linear estimation algorithms have been given in [48] to deal with uncertain observations, one-step delay and packet dropouts in a unified framework. On the other hand, by employing the linear matrix inequality technique, the design problems of optimal $H_{\infty}$ and $H_{2}$ filters have been investigated in [24], [42] for linear networked systems with multiple packet dropouts. Based on the quasi Markov-chain approach, the filtering algorithms have been given in [108] for linear networked systems in the simultaneous presence of random delay, packet dropouts and missing measurements. Besides, in [54], a robust filtering scheme has been provided for a class of linear time-varying systems with stochastic uncertainties, finite-step correlated process noises and missing measurements via the min-max game theory approach.

\section{B. Nonlinear Networked Systems}

As is well known, the nonlinearity is a ubiquitous feature existing in almost all practical systems that contributes significantly to the complexity of system modeling [89], [103], [109]-[112]. The occurrence of the nonlinearity would cause undesirable dynamic behaviors. Therefore, the filtering problems for general nonlinear networked systems have received considerable research attention and some useful methods have been given in [17], [35], [100], [113]-[116]. In terms of the Hamilton-Jacobi-Isaacs inequality method, the $H_{\infty}$ filtering problems have been investigated in [35], [100] for a general class of discrete-time nonlinear stochastic systems with missing measurements and random sensor delays, where sufficient criteria have been proposed to guarantee that the filtering error dynamics is stochastically stable irrespective of the presence of the missing measurements and random sensor delays. In [17], [113], [114], the extended Kalman filtering approaches have been given for general nonlinear networked systems with intermittent observations, state delay, and sensor failures, respectively. By using the Riccati equation method, the unscented Kalman filtering problems have been studied in [115], [116] for nonlinear networked systems 
with intermittent observations and packet dropout respectively, and sufficient conditions have been given to ensure the stochastic stability of the filtering error covariance, where the intermittent observations phenomenon in [115] is modeled by a Bernoulli random variable and the packet dropout phenomenon in [116] is characterized by a time-homogeneous Markov process.

In contrast to general nonlinearities, another class of nonlinearities (stochastic nonlinearities) deserves particular research attention since they occur randomly due probably to sudden environment changes, intermittent network congestion, changes in the interconnections of subsystems, random failures and repairs of the components, modification of the operating point of a linearized model of nonlinear systems [117]. Such stochastic nonlinearities include the state-dependent multiplicative noise disturbance as a special case. The filtering problems for networked systems with stochastic nonlinearities have already stirred some research interests and some latest results can be found in [16], [43], [45], [52], [101] based on several analysis techniques. For example, by using the Riccati-like difference equation approach, the extended Kalman filter has been designed in [16] for a class of time-varying networked systems with stochastic nonlinearities and multiple missing measurements. Moreover, the locally optimal Kalman-like filtering algorithms have been developed in [52], [101] for time-varying networked systems with stochastic nonlinearities, where the compensation schemes have been proposed to attenuate the effects from random sensor delays, random parameter matrices and gain-constraints onto the filtering performance. By using the recursive linear matrix inequality method, the robust $H_{\infty}$ filter has been constructed in [45] for a class of time-varying networked systems with stochastic nonlinearities and variance constraints. In [43], the filtering algorithm has been given for a class of discrete time-delay systems with stochastic nonlinearities by employing the semi-definite programme method.

Over the past two decades, as discussed in [118]-[120], the fuzzy-logic scheme has proven to be one of effective approaches for modeling the nonlinear networked systems. Therefore, the multi-objective filtering problems for nonlinear networked systems via the fuzzy method have gained considerable research attention. For example, based on the fuzzy interpolation method, a fuzzy stochastic partial differential system has been introduced in [121] to approximate the nonlinear stochastic partial differential system with random external disturbance and measurement noise, and a robust $H_{\infty}$ filtering algorithm has been developed by solving the linear matrix inequalities. In [57], a sequence of random variables obeying the Bernoulli distribution has been employed to model the phenomena of the randomly occurring uncertainties and the randomly occurring interval time-varying delays, and the fuzzy filtering problem has been studied for a class of nonlinear networked systems with channel fadings characterized by the Rice fading model. In addition, the intermittent measurements have been modeled in [44], [122] by using Bernoulli random variables with known occurrence probabilities and $H_{\infty}$ filtering algorithms have been developed for nonlinear networked systems based on the T-S fuzzy-model approach. In contrast to the modeling of the network-induced phenomena by using the Bernoulli probability distribution, a different modeling method has been introduced in [23], where the Markov chain has been used to model the random transmission delays and the $H_{2} / H_{\infty}$ filtering problem within fuzzy setting has been investigated for a class of nonlinear networked systems. Moreover, the event-triggered fuzzy filtering methods have been given in [123], [124] 
for nonlinear networked systems, where the developed filtering algorithms are capable of decreasing the communication load and energy consumption during the signal transmissions.

\section{Complex Networks and Sensor Networks}

Complex networks are composed of a group of interconnected nodes under certain topological structures [125], [126]. As is well known, the scale-free networks and small-world networks are two popular classes of complex networks characterized by the power-law degree distributions [127] and the short path lengths as well as high clustering [128]. During the past decade, the dynamical behavior analysis of the complex networks has become a very active research topic due to its application potentials in a wide range of realworld networks such as biological networks, computer networks, electrical power grids, cyber-physical systems, technological networks and social networks. Because of the importance and popularity of the complex networks, a rich body of research results has been published concerning various aspects of the network structure [129], [130]. Note that the system states are not always available in reality due to physical constraints, technological restrictions or expensive cost for measuring. Hence, it is also of great significance to estimate the states of the network nodes based on the available measurements. Accordingly, increasing research attention has been devoted to deal with the state estimation problems for time-invariant/time-varying complex networks with network-induced phenomena, see [131], [132] for some recent results.

On the other hand, the sensor networks equipped with distributed autonomous sensors have proven to be persistent research focuses which have gained an increasing attention in a variety of areas, and a great number of estimation schemes have been given in the literature [133]. It should be pointed out that the network-induced phenomena are inevitable in the sensor measurement outputs due to the noisy environment and limited communication capacity. The occurrence of the network-induced phenomena would greatly degrade the networked system performance or even lead to the divergence of the developed estimation schemes if not tackled properly. Hence, much work has been done on the topics of estimation, fusion, and distributed $H_{\infty}$ filtering for networked systems over sensor networks in [134]-[137] and the references therein. For example, the estimation and fusion problems have been studied for networked systems over sensor networks in [36], [136], [138], [139] with missing measurements, in [136], [139][141] with time-delays, in [142] with sensor saturations, in [143] with signal quantization, and in [144] with channel errors. We will return to the topics of estimation and fusion for complex networks/sensor networks later, and more details concerning the recent advances will be presented in the following section.

\section{LATEST PROGRESS}

Recently, the study on estimation, filtering and fusion for networked systems has attracted an increasing research interest and some important results have been reported in the literature. Here, we highlight some of the newest work, where the estimation, filtering and fusion algorithms have been presented to attenuate the effects from the network-induced phenomena onto the estimation performance under variance or $H_{\infty}$ constraints. 


\section{A. Filtering and Estimation for Networked Systems}

1) Filtering for Networked Systems: Recently, the modeling and filtering problems for time-varying systems have received increasing research attention owing to the fact that almost all real-world systems have certain parameters/structures that are time-varying. Therefore, some efficient filtering algorithms have been proposed for time-varying networked systems based on the Riccati-like difference equation approach or difference linear matrix inequality method. To mention a few, a Kalman-type filter has been designed in [52] for a class of time-varying nonlinear systems with random parameter matrices, correlated noises and fading measurements. Based on the result in [52], the recursive filtering problem has been investigated in [101] for time-varying nonlinear systems subject to finite-step correlated measurement noises, probabilistic sensor delays and gain-constraint. The developed filtering algorithm in [101] has the ability to attenuate the effects from the random sensor delays and gain-constraint onto the filtering performance and, moreover, it could be useful for addressing the gain-constrained issues arose in practical engineering with specified objectives, for example, to guarantee the unbiasedness property of the state estimates, simplify filter structure and handle the case of state estimates with linear equality constraint. In [145], the robust non-fragile filtering problem has been investigated for a class of linear time-varying systems subject to multiple packet dropouts and finite-step auto-correlated measurement noises, and a locally optimal filtering algorithm has been given. Subsequently, a globally optimal filtering scheme in the minimum mean-square error sense has been proposed in [146] by properly taking the statistical properties of correlated noises into account for the same addressed systems as in [145]. In [147], an optimal filtering algorithm has been given for linear time-varying system in the presence of the stochastic sensor gain degradations. Very recently, by using the backward Riccati equation method, an effective $H_{\infty}$ filtering scheme has been presented in [37] to handle the missing measurements and quantization effects in a same framework, and the developed result has been applied to address the mobile robot localization problem.

Parallel to the filtering problems for linear time-varying networked systems, the filtering problems for nonlinear time-varying networked systems have started to stir the initial research interest. For example, the recursive filtering problems have been studied in [16], [53] for two general classes of nonlinear networked time-varying systems with the multiple missing measurements and quantization measurements respectively, where some new recursive filtering algorithms have been developed by properly estimating the linearization error and based on the stochastic analysis technique. It has been shown that an optimal upper bound of the filtering error covariance can be obtained at each sampling instant by employing the filtering schemes in [16], [53]. In addition, more freedom degree and better filtering performance can be achieved by tuning the weight parameters, and the explicit forms of the filter parameters have been given in terms of the solutions to Riccati-like difference equations. Furthermore, a new non-fragile filter has been designed in [102] for a class of nonlinear time-varying networked systems with incomplete measurements consisting of the randomly occurring missing measurements and signal quantization, and a new filtering compensation algorithm has been given based on the Riccati-like difference equation approach. In addition, a probability-guaranteed $H_{\infty}$ finite-horizon filtering method has been proposed in [75] for a class of timevarying nonlinear systems with sensor saturations by utilizing difference linear matrix inequality technique, 
where the uniform distribution has been used to characterize the stochastic uncertainties in the system matrices and a new $H_{\infty}$ performance index with probability performance constraint has been introduced for time-varying systems in order to meet the specified engineering requirements. Very recently, in [148], the envelope-constrained $H_{\infty}$ filter has been constructed for a class of discrete time-varying networked systems with fading measurements and randomly occurring nonlinearities, where a novel envelope-constrained performance criterion over a finite horizon has been defined to further quantify the transient behavior of the filtering error.

2) State Estimation for Complex Networks: With respect to the state estimation problem for complex networks with network-induced phenomena, we mention some representative results as follows. In [149], the state estimator has been designed for an array of coupled discrete-time complex networks with discrete and distributed time delays. In [132], [150], the state estimation problems have been studied for complex networks with missing measurements, and sufficient criteria have been given to ensure the asymptotical stability of the estimation error in the mean-square sense by verifying the feasibility of certain linear matrix inequalities. The state estimation problem has been studied in [72] for a class of discrete nonlinear complex networks with randomly occurring phenomena, where the randomly occurring sensor saturations and randomly varying sensor delays have been addressed in a unified framework. In [151], the state estimation problem has been investigated for two-dimensional complex networks with randomly occurring nonlinearities and randomly varying sensor delays, where sufficient criteria have been given to guarantee the globally asymptotical stability of the two-dimensional estimation error dynamics in the mean square sense and the explicit expression of the estimator gains has also been provided. Based on the recursive linear matrix inequality approach, the state estimation algorithms have been given in [41], [152] for discrete time-varying complex networks. It is worth mentioning that, in [41], the authors have made the first attempt to discuss the uncertainties entering into the inner coupling matrix and introduce a new measurement model which can characterize the sensor saturations, signal quantization, and missing measurements in a unified framework. Very recently, in [153], the recursive state estimation problem has been investigated for an array of discrete time-varying coupled stochastic complex networks with missing measurements. By using the Riccati-like difference equations approach, new state estimation algorithm with covariance constraint has been developed for the first time and the estimator parameter has been characterized by the solutions to two Riccati-like difference equations.

\section{B. Distributed Filtering and Fusion for Networked Systems over Sensor Networks}

1) Distributed Estimation and Filtering for Networked Systems over Sensor Networks: In parallel to the recent developments of the networked control systems, in recent years, some initiatives have been made on the problems of distributed estimation and filtering for time-invariant/time-varying networked systems over sensor networks. Accordingly, several techniques have been proposed including linear matrix inequality method, recursive/parameter-dependent linear matrix inequality approach, and backward/forward Riccati difference equation method and so on. For example, by using the linear matrix inequality approach, a stochastic sampled-data scheme has been proposed in [154] to address the distributed filtering problem 
for time-invariant nonlinear systems over sensor networks, a distributed state estimator has been designed in [155] for discrete-time systems over sensor networks with randomly varying nonlinearities and missing measurements, and the distributed filters have been constructed in [25], [142] for nonlinear systems over sensor networks with randomly occurring saturations, quantization errors and successive packet dropouts. Besides, in [97], [99], the event-triggered distributed state estimation problems have been investigated for nonlinear systems over sensor networks with randomly occurring uncertainties, randomly occurring nonlinearities and packet dropouts.

Parallel to the distributed state estimation and filtering problems for time-invariant networked systems over sensor networks, the corresponding research for time-varying systems has gained the preliminary attention due to its engineering insights. By using the difference linear matrix inequality method, the $H_{\infty}$ filtering problems have been studied for time-varying systems over sensor networks in [156] with multiple missing measurements and in [46] with quantization errors as well as successive packet dropouts, where sufficient conditions have been given to ensure the pre-specified $H_{\infty}$ performance requirements by testing the feasibility of a set of linear matrix inequalities. By using the backward Riccati difference equation method, the distributed $H_{\infty}$ state estimation problem has been studied in [50] for a class of discrete time-varying nonlinear systems over sensor networks with stochastic parameters and stochastic nonlinearities, and a necessary and sufficient condition has been given to ensure the pre-defined $H_{\infty}$ performance constraint. In [157], a distributed filter has been designed for a class of linear discrete timevarying stochastic systems via event-based communication mechanism, and a locally optimal distributed filtering algorithm has been given based on the forward Riccati difference equation approach which is suitable for online applications.

2) Multi-Sensor Fusion for Networked Systems: As mentioned above, the multi-sensor data fusion algorithms can be generally classified into two types: centralized fusion and distributed fusion algorithms. In this section, some new multi-sensor fusion schemes based on different weighted fusion mechanisms for networked systems are reviewed. In [158], by using the innovation analysis technique and augmentation approach, the optimal centralized fusion estimators (including filter, predictor and smoother) in the minimum variance sense have been designed for a class of linear discrete time-varying stochastic systems with random delays, packet dropouts and uncertain observations, where the stability of the developed estimation algorithms has been discussed and sufficient criterion has been given to verify the existence of the centralized fusion steady-state estimators. Recently, by employing similar technique as in [158], the optimal centralized and distributed fusion estimation problems have been addressed in [159] for linear discrete time-varying multi-sensor system with different packet dropout rates, and the centralized fusion estimators (including filter, predictor and smoother) in the linear minimum variance sense have been firstly designed and, subsequently, the distributed fusion estimation algorithm based on the scalar-weighted fusion mechanism has also been provided in order to decrease the computational cost and improve the reliability.

On the other hand, according to the matrix-weighted fusion mechanism, several distributed fusion algorithms have been developed in order to improve the fault-tolerance ability [160]-[163]. To be more specific, the Kalman-like distributed fusion filters (one-step predictors) have been constructed in [160] 
for linear multi-sensor time-varying stochastic system in the simultaneous presence of parameter uncertainties, missing measurements and unknown measurement disturbances, and the optimal filter gains have been obtained based on the linear unbiased minimum variance criterion. In [161], the distributed fusion estimation algorithm has been given for linear discrete time-varying stochastic systems with multi-sensor missing observations, where the case of the finite-step observations missing has been discussed. Moreover, a multi-sensor distributed fusion estimation algorithm has been developed in [162] for networked systems, where the measurements of all sensors are transmitted individually over different communication channels with individual random delay and packet dropout rates. Besides, when there exist the auto-correlated and cross-correlated noises, a robust distributed weighted Kalman filter fusion method has been presented in [163] for a class of uncertain time-varying systems with stochastic uncertainties without resorting the state augmentation method. By using the projection theory, an optimal fusion algorithm has been given in [164] for a class of multi-sensor stochastic singular systems with multiple state delays and measurement delays.

In [165], based on the federated filtering algorithm, a novel networked multi-sensor data-fusion scheme has been proposed to deal with the effects from both the packet losses and the transmission delays. A globally optimal distributed Kalman filtering fusion method has been proposed in [166] for a class of timevarying systems, where the developed fusion algorithm has the advantage to decrease the computational burden and address the case when the filtering error covariance is singular. For the case that the state-space model of the signal is unavailable, both distributed and centralized fusion schemes have been developed in [167] to deal with the phenomena of the multi-sensor random measurement delays which are modeled by the homogeneous Markov chains and, subsequently, the extended result has been given in [168] to handle the missing measurements and random measurement delays with individual delay rate in a unified framework. Moreover, the distributed Kalman filtering fusion problems have been studied in [38], [169] for networked systems with missing measurements, random transmission delays and packet dropouts, new distributed fusion Kalman filters have been designed based on the innovation analysis method and matrixweighted fusion mechanism. With respect to the multi-sensor fusion for nonlinear networked systems, a few results can be found in the literature. In [170], the centralized and distributed $H_{\infty}$ fusion filters have been designed for a class of discrete nonlinear stochastic systems with time-invariant delay and missing measurements. It has been shown that, for both missing measurements and time-delay, the fusion error in [170] is globally asymptotically stable in the mean-square sense and the prescribed $H_{\infty}$ performance can be achieved.

\section{CONClusions And Future Works}

In this paper, we have reviewed some recent advances on estimation, filtering and fusion for timeinvariant/time-varying stochastic networked systems. Firstly, the developments of the network-induced phenomena have been surveyed. Secondly, the analysis and synthesis of the networked systems have been discussed, where the linear/nonlinear networked systems, complex networks and sensor networks with network-induced phenomena have been mainly summarized. Subsequently, some recent advances 
on estimation, filtering and fusion for networked systems have been reviewed. In particular, the multiobjective filtering algorithms (involving variance constraint, $H_{\infty}$ performance requirement, and probability performance constraint) have been surveyed for time-varying nonlinear networked systems. Based on the literature review, some related topics for further research work can be listed as follows.

- The estimation and filtering problems for networked systems with more general nonlinearities would be one of future research topics, especially when both variance constraint and multiple networkinduced phenomena are considered simultaneously.

- The distributed filtering problem for networked systems is of engineering significance, especially when it comes to the distributed filtering problem for time-varying nonlinear networked systems. Hence, the design of distributed filter for time-varying nonlinear networked systems would be an interesting research direction.

- The multi-sensor fusion problem for nonlinear networked systems would be a challenging research topic.

- A potential trend for future research is to generalize the current methods to tackle the estimation and filtering problems for nonlinear networked systems under the event-triggered mechanism.

- Another interesting research direction is to address the estimation and filtering problems for nonlinear networked systems under different communication protocols (round-robin protocol and try-oncediscard protocol).

- The performance analysis of the estimation/filtering algorithm constitutes one of future research topics, such as the convergence of the developed algorithm and the monotonicity/sensibility with respect to the statistical information of the network-induced phenomena.

\section{REFERENCES}

[1] K. Han, J. Luo, Y. Liu, and A. V. Vasilakos, Algorithm design for data communications in duty-cycled wireless sensor networks: a survey, IEEE Communications Magazine, vol. 51, no. 7, pp. 107-113, 2013.

[2] J. Baillieul and P. J. Antsaklis, Control and communication challenges in networked real-time systems, Proceedings of the IEEE, vol. 95, no. 1, pp. 9-28, 2007.

[3] W. P. M. H. Heemels, A. R. Teel, N. van de Wouw, and D. Nešić, Networked control systems with communication constraints: tradeoffs between transmission intervals, delays and performance, IEEE Transactions on Automatic Control, vol. 55, no. 8, pp. 1781-1796, 2010.

[4] J. Hu, Z. Wang, H. Dong, and H. Gao, Recent advances on recursive filtering and sliding mode design for networked nonlinear stochastic systems: a survey, Mathematical Problems in Engineering, vol. 2013, Article ID 646059, DOI: 10.1155/2013/646059, 12 pages, 2013.

[5] X. Shen, E. Song, Y. Zhu, and Y. Luo, Globally optimal distributed Kalman fusion with local out-of-sequence-measurement updates, IEEE Transactions on Automatic Control, vol. 54, no. 8, pp. 1928-1934, 2009.

[6] H. Gao and T. Chen, Network-based $H_{\infty}$ output tracking control, IEEE Transactions on Automatic Control, vol. 53, no. 3, pp. 655-667, 2008.

[7] J. Hu, Z. Wang, and H. Gao, Nonlinear Stochastic Systems with Network-Induced Phenomena: Recursive Filtering and Sliding-Mode Design, Springer, London, UK, 2015.

[8] M. V. Basin, J. J. Maldonado, and H. R. Karimi, Mean-square filtering for polynomial system states confused with poisson noises over polynomial observations, Modelling Identification and Control, vol. 32, no. 2, pp. 47-55, 2011.

[9] M. V. Basin, A. G. Loukianov, and M. Hernandez-Gonzalez, Joint state and parameter estimation for uncertain stochastic nonlinear polynomial systems, International Journal of Systems Science, vol. 44, no. 7, pp. 1200-1208, 2013. 
[10] R. E. Kalman, A new approach to linear filtering and prediction problems, Journal of Fluids Engineering, vol. 82, no. 1, pp. 35-45, 1960.

[11] B. Safarinejadian and E. Kowsari, Fault detection in non-linear systems based on GP-EKF and GP-UKF algorithms, Systems Science and Control Engineering: An Open Access Journal, vol. 2, no. 1, pp. 610-620, 2014.

[12] H. Lv, G. Wei, Z. Ding, and X. Ding, Sensorless control for the brushless DC motor: an unscented Kalman filter algorithm, Systems Science and Control Engineering: An Open Access Journal, vol. 3, no. 1, pp. 8-13, 2015.

[13] D. Li, S. Kar, F. E. Alsaadi, and S. Cui, Distributed Kalman filtering with quantized sensing state, IEEE Transactions on Signal Processing, vol. 63, no. 19, pp. 5180-5193, 2015.

[14] B. Safarinejadian and M. Yousefi, Static alignment of inertial navigation systems using an adaptive multiple fading factors Kalman filter, Systems Science and Control Engineering: An Open Access Journal, vol. 3, no. 1, pp. 351-359, 2015.

[15] H. Ahmada and T. Namerikawa, Extended Kalman filter-based mobile robot localization with intermittent measurements, Systems Science and Control Engineering: An Open Access Journal, vol. 1, no. 1, pp. 113-126, 2013.

[16] J. Hu, Z. Wang, H. Gao, and L. K. Stergioulas, Extended Kalman filtering with stochastic nonlinearities and multiple missing measurements, Automatica, vol. 48, no. 9, pp. 2007-2015, 2012.

[17] S. Kluge, K. Reif, and M. Brokate, Stochastic stability of the extended Kalman filter with intermittent observations, IEEE Transactions on Automatic Control, vol. 55, no. 2, pp. 514-518, 2010.

[18] A. Chatterjee, R. Fournier, A. Nait-Ali, and P. Siarry, A postural information-based biometric authentication system employing Stransform, radial basis function network, and extended Kalman filtering, IEEE Transactions on Instrumentation and Measurement, vol. 59, no. 12, pp. 3131-3138, 2010.

[19] T. M. Cheng, V. Malyavej, and A. V. Savkin, Decentralized robust set-valued state estimation in networked multiple sensor systems, Computers \& Mathematics with Applications, vol. 59, no. 8, pp. 2636-2646, 2010.

[20] D. Shi, T. Chen, and L. Shi, On set-valued Kalman filtering and its application to event-based state estimation, IEEE Transactions on Automatic Control, vol. 60, no. 5, pp. 1275-1290, 2015.

[21] G. Wei, S. Liu, Y. Song, and Y. Liu, Probability-guaranteed set-membership filtering for systems with incomplete measurements, Automatica, vol. 60, pp. 12-16, 2015.

[22] H. Gao, J. Lam, L. Xie, and C. Wang, New approach to mixed $H_{2} / H_{\infty}$ filtering for polytopic discrete-time systems, IEEE Transactions on Signal Processing, vol. 53, no. 8, pp. 3183-3192, 2005.

[23] M. Liu, X. Liu, Y. Shi, and S. Wang, T-S fuzzy-model-based $H_{2}$ and $H_{\infty}$ filtering for networked control systems with two-channel Markovian random delays, Digital Signal Processing, vol. 27, pp. 167-174, 2014.

[24] M. Sahebsara, T. Chen, and S. L. Shah, Optimal $H_{2}$ filtering in networked control systems with multiple packet dropout, IEEE Transactions on Automatic Control, vol. 52, no. 8, pp. 1508-1513, 2007.

[25] H. Dong, Z. Wang, and H. Gao, Distributed $H_{\infty}$ filtering for a class of Markovian jump nonlinear time-delay systems over lossy sensor networks, IEEE Transactions on Industrial Electronics, vol. 60, no. 10, pp. 4665-4672, 2013.

[26] B. Shen, Z. Wang, Y. S. Hung, and G. Chesi, Distributed $H_{\infty}$ filtering for polynomial nonlinear stochastic systems in sensor networks, IEEE Transactions on Industrial Electronics, vol. 58, no. 5, pp. 1971-1979, 2011.

[27] B. Shen, Z. Wang, and Y. S. Hung, Distributed $H_{\infty}$-consensus filtering in sensor networks with multiple missing measurements: the finite-horizon case, Automatica, vol. 46, no. 10, pp. 1682-1688, 2010.

[28] S. S. Kia, J. Cortes, and S. Martinez, Distributed event-triggered communication for dynamic average consensus in networked systems, Automatica, vol. 59, pp. 112-119, 2015.

[29] R. Caballero-Águila, A. Hermoso-Carazo, and J. Linares-Pérez, Optimal state estimation for networked systems with random parameter matrices, correlated noises and delayed measurements, International Journal of General Systems, vol. 44, no. 2, pp. 142-154, 2015.

[30] S. Sun, L. Xie, W. Xiao, and Y. C. Soh, Optimal linear estimation for systems with multiple packet dropouts, Automatica, vol. 44, no. 5, pp. 1333-1342, 2008.

[31] S. Sun, Linear minimum variance estimators for systems with bounded random measurement delays and packet dropouts, Signal Processing, vol. 89, no. 7, pp. 1457-1466, 2009.

[32] S. Sun and W. Xiao, Optimal linear estimators for systems with multiple random measurement delays and packet dropouts, International Journal of Systems Science, vol. 44, no. 2, pp. 358-370, 2013.

[33] B. Khaleghi, A. Khamis, F. O. Karray, and S. N. Razavi, Multisensor data fusion: a review of the state-of-the-art, Information Fusion, vol. 14, no. 1, pp. 28-44, 2013. 
[34] D. Ding, Z. Wang, F. E. Alsaadi, and B. Shen, Receding horizon filtering for a class of discrete time-varying nonlinear systems with multiple missing measurements, International Journal of General Systems, vol. 44, no. 2, pp. 198-211, 2015.

[35] B. Shen, Z. Wang, H. Shu, and G. Wei, On nonlinear $H_{\infty}$ filtering for discrete-time stochastic systems with missing measurements, IEEE Transactions on Automatic Control, vol. 53, no. 9, pp. 2170-2180, 2008.

[36] H. Geng, Y. Liang, and X. Zhang, Linear-minimum-mean-square-error observer for multi-rate sensor fusion with missing measurements, IET Control Theory and Applications, vol. 8, no. 14, pp. 1375-1383, 2014.

[37] Z. Wang, H. Dong, B. Shen, and H. Gao, Finite-horizon $H_{\infty}$ filtering with missing measurements and quantization effects, IEEE Transactions on Automatic Control, vol. 58, no. 7, pp. 1707-1718, 2013.

[38] B. Chen, W. Zhang, and L. Yu, Distributed fusion estimation with missing measurements, random transmission delays and packet dropouts, IEEE Transactions on Automatic Control, vol. 59, no. 7, pp. 1961-1967, 2014.

[39] S. Sun and G. Wang, Modeling and estimation for networked systems with multiple random transmission delays and packet losses, Systems \& Control Letters, vol. 73, pp. 6-16, 2014.

[40] Z. Wang, B. Shen, and X. Liu, $H_{\infty}$ filtering with randomly occurring sensor saturations and missing measurements, Automatica, vol. 48, no. 3, pp. 556-562, 2012.

[41] B. Shen, Z. Wang, D. Ding, and H. Shu, $H_{\infty}$ state estimation for complex networks with uncertain inner coupling and incomplete measurements, IEEE Transactions on Neural Networks and Learning Systems, vol. 24, no. 12, pp. 2027-2037, 2013.

[42] M. Sahebsara, T. Chen, and S. L. Shah, Optimal $H_{\infty}$ filtering in networked control systems with multiple packet dropouts, Systems \& Control Letters, vol. 57, no. 9, pp. 696-702, 2008.

[43] G. Wei, Z. Wang, and H. Shu, Robust filtering with stochastic nonlinearities and multiple missing measurements, Automatica, vol. 45, no. 3, pp. 836-841, 2009.

[44] H. Gao, Y. Zhao, J. Lam, K. Chen, $H_{\infty}$ fuzzy filtering of nonlinear systems with intermittent measurements, IEEE Transactions on Fuzzy Systems, vol. 17, no. 2, pp. 291-300, 2009.

[45] H. Dong, Z. Wang, D. W. C. Ho, and H. Gao, Variance-constrained $H_{\infty}$ filtering for a class of nonlinear time-varying systems with multiple missing measurements: the finite-horizon case, IEEE Transactions on Signal Processing, vol. 58, no. 5, pp. 2534-2543, 2010.

[46] H. Dong, Z. Wang, and H. Gao, Distributed filtering for a class of time-varying systems over sensor networks with quantization errors and successive packet dropouts, IEEE Transactions on Signal Processing, vol. 60, no. 6, pp. 3164-3173, 2012.

[47] R. Caballero-Águila, A. Hermoso-Carazo, J. D. Jiménez-López, J. Linares-Pérez, and S. Nakamori, Signal estimation with multiple delayed sensors using covariance information, Digital Signal Processing, vol. 20, no. 2, pp. 528-540, 2010.

[48] R. Caballero-Águila, A. Hermoso-Carazo, and J. Linares-Pérez, Covariance-based estimation algorithms in networked systems with mixed uncertainties in the observations, Signal Processing, vol. 94, pp. 163-173, 2014.

[49] D. Ding, Z. Wang, J. Lam, and B. Shen, Finite-horizon $H_{\infty}$ control for discrete time-varying systems with randomly occurring nonlinearities and fading measurements, IEEE Transactions on Automatic Control, vol. 60, no. 9, pp. 2488-2493, 2015.

[50] D. Ding, Z. Wang, H. Dong, and H. Shu, Distributed $H_{\infty}$ state estimation with stochastic parameters and nonlinearities through sensor networks: the finite-horizon case, Automatica, vol. 48, no. 8, pp. 1575-1585, 2012.

[51] S. Dey, A. S. Leong, and J. S. Evans, Kalman filtering with faded measurements, Automatica, vol. 45, no. 10, pp. 2223-2233, 2009.

[52] J. Hu, Z. Wang, and H. Gao, Recursive filtering with random parameter matrices, multiple fading measurements and correlated noises, Automatica, vol. 49, no. 11, pp. 3440-3448, 2013.

[53] J. Hu, Z. Wang, B. Shen, and H. Gao, Quantized recursive filtering for a class of nonlinear systems with multiplicative noises and missing measurements, International Journal of Control, vol. 86, no. 4, pp. 650-663, 2013.

[54] J. Feng, Z. Wang, and M. Zeng, Recursive robust filtering with finite-step correlated process noises and missing measurements, Circuits Systems and Signal Processing, vol. 30, no. 6, pp. 1355-1368, 2011.

[55] A. S. Leong, S. Dey, G. N. Nair, and P. Sharma, Power allocation for outage minimization in state estimation over fading channels, IEEE Transactions on Signal Processing, vol. 59, no. 7, pp. 3382-3397, 2008.

[56] N. Elia, Remote stabilization over fading channels, Systems \& Control Letter, vol. 54, no. 3, pp. 237-249, 2005.

[57] S. Zhang, Z. Wang, D. Ding, and H. Shu, Fuzzy filtering with randomly occurring parameter uncertainties, interval delays, and channel fadings, IEEE Transactions on Cybernetics, vol. 44, no. 3, pp. 406-417, 2014.

[58] R. W. Brockett and D. Liberzon, Quantized feedback stabilization of linear systems, IEEE Transactions on Automatic Control, vol. 45, no. 7, pp. 1279-1289, 2000.

[59] H. Dong, Z. Wang, S. X. Ding, and H. Gao, Finite-horizon reliable control with randomly occurring uncertainties and nonlinearities subject to output quantization, Automatica, vol. 52, pp. 355-362, 2015. 
[60] H. Gao and T. Chen, A new approach to quantized feedback control systems, Automatica, vol. 44, no. 2, pp. 534-542, 2008.

[61] D. Liberzon, Hybrid feedback stabilization of systems with quantized signals, Automatica, vol. 39, no. 9, pp. 1543-1554, 2003.

[62] K. You and L. Xie, Minimum data rate for mean square stabilization of discrete LTI systems over lossy channels, IEEE Transactions on Automatic Control, vol. 55, no. 10, pp. 2373-2378, 2010.

[63] Y. Sharon, and D. Liberzon, Input to state stabilizing controller for systems with coarse quantization, IEEE Transactions on Automatic Control, vol. 57, no. 4, pp. 830-844, 2012.

[64] A. J. Rojas and F. Lotero, Signal-to-noise ratio limited output feedback control subject to channel input quantization, IEEE Transactions on Automatic Control, vol. 60, no. 2, pp. 475-479, 2015.

[65] N. Elia and S. Mitter, Stabilization of linear systems with limited information, IEEE Transactions on Automatic Control, vol. 46, no. 9, pp. 1384-1400, 2001.

[66] M. Fu and L. Xie, The sector bound approach to quantized feedback control, IEEE Transactions on Automatic Control, vol. 50, no. 11, pp. 1698-1711, 2005.

[67] M. L. Corradini and G. Orlando, Robust quantized feedback stabilization of linear systems, Automatica, vol. 44, no. 9, pp. 2458-2462, 2008.

[68] K. You, W. Sun, M. Fu, and L. Xie, Attainability of the minimum data rate for stabilization of linear systems via logarithmic quantization, Automatica, vol. 47, no. 1, pp. 170-176, 2011.

[69] J. You, S. Yin, and H. Gao, Fault detection for discrete systems with network-induced nonlinearities, IEEE Transactions on Industrial Informatics, vol. 10. no. 4, pp. 2216-2223, 2014.

[70] F. Li, P. Shi, X. Wang, and R. Agarwal, Fault detection for networked control systems with quantization and Markovian packet dropouts, Signal Processing, vol. 111, pp. 106-112, 2015.

[71] H. Dong, Z. Wang, and H. Gao, Fault detection for Markovian jump systems with sensor saturations and randomly varying nonlinearities, IEEE Transactions on Circuits and Systems-I: Regular Papers, vol. 59, no. 10, pp. 2354-2362, 2012.

[72] D. Ding, Z. Wang, B. Shen, and H. Shu, $H_{\infty}$ state estimation for discrete-time complex networks with randomly occurring sensor saturations and randomly varying sensor delays, IEEE Transactions on Neural Networks and Learning Systems, vol. 23, no. 5, pp. 725-736, 2012.

[73] Y. Xiao, Y. Cao, and Z. Lin, Robust filtering for discrete-time systems with saturation and its application to transmultiplexers, IEEE Transactions on Signal Processing, vol. 52, no. 5, pp. 1266-1277, 2004.

[74] F. Yang and Y. Li, Set-membership filtering for systems with sensor saturation, Automatica, vol. 45, no. 8, pp. 1896-1902, 2009.

[75] J. Hu, Z. Wang, H. Gao, and L. K. Stergioulas, Probability-guaranteed $H_{\infty}$ finite-horizon filtering for a class of nonlinear time-varying systems with sensor saturation, System \& Control Letters, vol. 61, no. 4, pp. 477-484, 2012.

[76] J. Liang, Z. Wang, and X. Liu, On passivity and passification of stochastic fuzzy systems with delays: the discrete-time case, IEEE Transactions on Systems, Man, and Cybernetics-Part B: Cybernetics, vol. 40, no. 3, pp. 964-969, 2010.

[77] J. Hu, D. Chen, and J. Du, State estimation for a class of discrete nonlinear systems with randomly occurring uncertainties and distributed sensor delays, International Journal of General Systems, vol. 43, no. 3-4, pp. 387-401, 2014.

[78] S. Yin, H. Luo, and S. Ding, Real-time implementation of fault-tolerant control systems with performance optimization, IEEE Transactions on Industrial Electronics, vol. 64, no. 5, pp. 2402-2411, 2014.

[79] J. Liang and J. Cao, Global output convergence of recurrent neural networks with distributed delays, Nonlinear Analysis-Real World Applications, vol. 8, no. 1, pp. 187-197, 2007.

[80] Y. Zhang, Z. Liu, H. Fang, and H. Chen, $H_{\infty}$ fault detection for nonlinear networked systems with multiple channels data transmission pattern, Information Sciences, vol. 221, pp. 534-543, 2013.

[81] Y. Liu, Y. Wang, X. Zhu, and X. Liu, Optimal guaranteed cost control of a class of hybrid systems with mode-dependent mixed time delays, International Journal of Systems Science, vol. 45, no. 7, pp. 1528-1538, 2014.

[82] Y. Luo, G. Wei, Y. Liu, and X. Ding, Reliable $H_{\infty}$ state estimation for 2-D discrete systems with infinite distributed delays and incomplete observations, International Journal of General Systems, vol. 44, no. 2, pp. 155-168, 2015.

[83] G. Wei, F. Han, L. Wang, and Y. Song, Reliable $H_{\infty}$ filtering for discrete piecewise linear systems with infinite distributed delays, International Journal of General Systems, vol. 43, no. 3-4, pp. 346-358, 2014.

[84] S. Xu and J. Lam, A survey of linear matrix inequality techniques in stability analysis of delay systems, International Journal of Systems Science, vol. 39, no. 12, pp. 1095-1113, 2008.

[85] Y. S. Moon, P. Park, W. H. Kwon, and Y. S. Lee, Delay-dependent robust stabilization of uncertain state-delayed systems, International Journal of Control, vol. 74, no. 14, pp. 1447-1455, 2001. 
[86] E. Fridman and U. Shaked, A descriptor system approach $H_{\infty}$ control of linear time-delay systems, IEEE Transactions on Automatic Control, vol. 47, no. 2, pp. 253-270, 2002.

[87] M. Wu, Y. He, J. She, and G. Liu, Delay-dependent criteria for robust stability of time-varying delay systems, Automatica, vol. 40, no. 8, pp. 1435-1439, 2004.

[88] D. Peaucelle, D. Arzelier, D. Henrion, and F. Gouaisbaut, Quadratic separation for feedback connection of an uncertain matrix and an implicit linear transformation, Automatica, vol. 43, no. 5, pp. 795-804, 2007.

[89] J. Hu, Z. Wang, H. Gao, and L. K. Stergioulas, Robust sliding mode control for discrete stochastic systems with mixed time-delays, randomly occurring uncertainties and randomly occurring nonlinearities, IEEE Transactions on Industrial Electronics, vol. 59, no. 7, pp. 3008-3015, 2012.

[90] H. Dong, Z. Wang, and H. Gao, Robust $H_{\infty}$ filtering for a class of nonlinear networked systems with multiple stochastic communication delays and packet dropouts, IEEE Transactions on Signal Processing, vol. 58, no. 4, pp. 1957-1966, 2010.

[91] C. Han, H. Zhang, and M. Fu, Optimal filtering for networked systems with Markovian communication delays, Automatica, vol. 49, no. 10, pp. 3097-3104, 2013.

[92] S. Sun and J. Ma, Linear estimation for networked control systems with random transmission delays and packet dropouts, Information Sciences, vol. 269, pp. 349-365, 2014.

[93] D. Chen, L. Xu, and J. Du, Optimal filtering for systems with finite-step autocorrelated process noises, random one-step sensor delay and missing measurements, Communications in Nonlinear Science and Numerical Simulation, vol. 32, pp. 211-224, 2016.

[94] H Dong, Z. Wang, S. X. Ding, and H. Gao, Finite-horizon reliable control with randomly occurring uncertainties and nonlinearities subject to output quantization, Automatica, vol. 52, pp. 355-362, 2015.

[95] J. Hu, S. Liu, D. Ji, and S. Li, On co-design of filter and fault estimator against randomly occurring nonlinearities and randomly occurring deception attacks, International Journal of General Systems, DOI: 10.1080/03081079.2015.1106730, in press, 2016.

[96] Y. Liu, F. E. Alsaadi, X. Yin, and Y. Wang, Robust $H_{\infty}$ filtering for discrete nonlinear delayed stochastic systems with missing measurements and randomly occurring nonlinearities, International Journal of General Systems, vol. 44, no. 2, pp. 169-181, 2015.

[97] J. Hu, Z. Wang, J. Liang, and H. Dong, Event-triggered distributed state estimation with randomly occurring uncertainties and nonlinearities over sensor networks: a delay-fractioning approach, Journal of the Franklin Institute, vol. 352, no. 9, pp. 3750-3763, 2015.

[98] J. Hu, Z. Wang, and H. Gao, A delay-fractioning approach to robust sliding mode control for discrete-time stochastic systems with randomly occurring nonlinearities, IMA Journal of Mathematical Control and Information, vol. 28, no. 3, pp. 345-363, 2011.

[99] D. Ding, Z. Wang, B. Shen, and H. Dong, Event-triggered distributed $H_{\infty}$ state estimation with packet dropouts through sensor networks, IET Control Theory \& Applications, vol. 9, no. 13, pp. 1948-1955, 2015.

[100] B. Shen, Z. Wang, H. Shu, and G. Wei, $H_{\infty}$ filtering for nonlinear discrete-time stochastic systems with randomly varying sensor delays, Automatica, vol. 45, no. 4, pp. 1032-1037, 2009.

[101] J. Hu, Z. Wang, B. Shen, and H. Gao, Gain-constrained recursive filtering with stochastic nonlinearities and probabilistic sensor delays, IEEE Transactions on Signal Processing, vol. 61, no. 5, pp. 1230-1238, 2013.

[102] J. Hu, J. Liang, D. Chen, D. Ji, and J. Du, A recursive approach to non-fragile filtering for networked systems with stochastic uncertainties and incomplete measurements, Journal of the Franklin Institute, vol. 352, no. 5, pp. 1946-1962, 2015.

[103] H. Dong, Z. Wang, S. X. Ding, and H. Gao, Finite-horizon estimation of randomly occurring faults for a class of nonlinear time-varying systems, Automatica, vol. 50, no. 12, pp. 3182-3189, 2014.

[104] D. Ding, Z. Wang, and B. Shen, Recent advances on distributed filtering for stochastic systems over sensor networks, International Journal of General Systems, vol. 43, no. 3-4, pp. 372-386, 2014.

[105] H. Dong, Z. Wang, F. E. Alsaadi, and B. Ahmad, Event-triggered robust distributed state estimation for sensor networks with statedependent noises, International Journal of General Systems, vol. 44, no. 2, pp. 254-266, 2015.

[106] Q. Li, B. Shen, J. Liang, and H. Shu, Event-triggered synchronization control for complex networks with uncertain inner coupling, International Journal of General Systems, vol. 44, no. 2, pp. 212-225, 2015.

[107] L. Sheng, W. Zhang, and M. Gao, Relationship between Nash equilibrium strategies and $H_{2} / H_{\infty}$ control of stochastic Markov jump systems with multiplicative noise, IEEE Transactions on Automatic Control, vol. 59, no. 9, pp. 2592-2597, 2014.

[108] M. Moayedi, Y. K. Foo, Y. C. Soh, Optimal and suboptimal minimum-variance filtering in networked systems with mixed uncertainties of random sensor delays, packet dropouts and missing measurements, International Journal of Control Automation and Systems, vol. 8 , no. 6, pp. 1179-1188, 2010. 
[109] D. Ding, Z. Wang, J. Hu, and H. Shu, Dissipative control for state-saturated discrete time-varying systems with randomly occurring nonlinearities and missing measurements, International Journal of Control, vol. 86, no. 4, pp. 674-688, 2013.

[110] J. Hu, Z. Wang, Y. Niu, and L. K. Stergioulas. $H_{\infty}$ sliding mode observer design for a class of nonlinear discrete time-delay systems: a delay-fractioning approach, International Journal of Robust and Nonlinear Control, vol. 22, no. 16, pp. 1806-1826, 2012.

[111] H. Li, H. Gao, P. Shi, and X. Zhao, Fault-tolerant control of Markovian jump stochastic systems via the augmented sliding mode observer approach, Automatica, vol. 50, no. 7, pp. 1825-1834, 2014.

[112] L. Wang, G. Wei, and W. Li, Probability-dependent $H_{\infty}$ synchronization control for dynamical networks with randomly varying nonlinearities, Neurocomputing, vol. 133, pp. 369-376, 2014.

[113] A. Gopalakrishnan, N. S. Kaisare, and S. Narasimhan, Incorporating delayed and infrequent measurements in extended Kalman filter based nonlinear state estimation, Journal of Process Control, vol. 21, no. 1, pp. 119-129, 2011.

[114] X. Wang and E. E. Yaz, Stochastically resilient extended Kalman filtering for discrete-time nonlinear systems with sensor failures, International Journal of Systems Science, vol. 45, no. 7, pp. 1393-1401, 2014.

[115] L. Li and Y. Xia, Stochastic stability of unscented Kalman filter with intermittent observations, Automatica, vol. 48, no. 5, pp. 978-981, 2012.

[116] L. Li and Y. Xia, Unscented Kalman filter over unreliable communication networks with Markovian packet dropouts, IEEE Transactions on Automatic Control, vol. 58, no. 12, pp. 3224-3230, 2013.

[117] J. Hu, Z. Wang, H. Gao, and L. K. Stergioulas, Robust $H_{\infty}$ sliding mode control for discrete time-delay systems with stochastic nonlinearities, Journal of the Franklin Institute, vol. 349, no. 4, pp. 1459-1479, 2012.

[118] Y. Niu, D. W. C. Ho, and C. Li, Filtering for discrete fuzzy stochastic systems with sensor nonlinearities, IEEE Transactions on Fuzzy Systems, vol. 18, no. 5, pp. 971-978, 2010.

[119] D. Peng, C. Gao, and Z. Gao, Generalized hesitant fuzzy synergetic weighted distance measures and their application to multiple criteria decision-making, Applied Mathematical Modelling, vol. 37, no. 8, pp. 5837-5850, 2013.

[120] L. Sheng, M. Gao, W. Zhang, and B. S. Chen, Infinite horizon $H_{\infty}$ control for nonlinear stochastic Markov jump systems with $(x, u, v)$-dependent noise via fuzzy approach, Fuzzy Sets and Systems, vol. 273, pp. 105-123, 2015.

[121] B. Chen, W. Chen, and W. Zhang, Robust filter for nonlinear stochastic partial differential systems in sensor signal processing: fuzzy approach, IEEE Transactions on Fuzzy Systems, vol. 20, no. 5, pp. 957-970, 2012.

[122] B. Jiang, Z. Mao, and P. Shi, $H_{\infty}$-filter design for a class of networked control systems via T-S fuzzy-model approach, IEEE Transactions on Fuzzy Systems, vol. 18, no. 1, pp. 201-208, 2010.

[123] J. Zhang and C. Peng, Event-triggered $H_{\infty}$ filtering for networked Takagi-Sugeno fuzzy systems with asynchronous constraints, IET Signal Processing, vol. 9, no. 5, pp. 403-411, 2015.

[124] H. Wang, P. Shi, J. Zhang, Event-triggered fuzzy filtering for a class of nonlinear networked control systems, Signal Processing, vol. 113, pp. 159-168, 2015.

[125] J. Wang, H. Wu, T. Huang, and S. Ren, Passivity and synchronization of linearly coupled reaction-diffusion neural networks with adaptive coupling, IEEE Transactions on Cybernetics, vol. 45, no. 9, pp. 1942-1952, 2015.

[126] L. Wang, G. Wei, and H. Shu, State estimation for complex networks with randomly occurring coupling delays, Neurocomputing, vol. 122, pp. 513-520, 2013.

[127] A. L. Barabási and R. Albert, Emergence of scaling in random networks, Science, vol. 286, no. 5439, pp. 509-512, 1999.

[128] S. H. Strogatz and D. J. Watts, Collective dynamics of 'small-world' networks, Nature, vol. 393, no. 6684, pp. 440-442, 1998.

[129] J. Lu and D. W. C. Ho, Globally exponential synchronization and synchronizability for general dynamical networks, IEEE Transactions on Systems, Man, and Cybernetics-Part B: Cybernetics, vol. 40, no. 2, pp. 350-361, 2010.

[130] Z. Wang, Y. Wang, and Y. Liu, Global synchronization for discrete-time stochastic complex networks with randomly occurred nonlinearities and mixed time delays, IEEE Transactions on Neural Networks, vol. 21, no. 1, pp. 11-25, 2010.

[131] H. Huang, G. Feng, and J. Cao, Robust state estimation for uncertain neural networks with time-varying delay, IEEE Transactions on Neural Networks, vol. 19, no. 8, pp. 1329-1339, 2008.

[132] J. Liang, Z. Wang, and X. Liu, State estimation for coupled uncertain stochastic networks with missing measurements and time-varying delays: the discrete-time case, IEEE Transactions on Neural Networks, vol. 20, no. 5, pp. 781-793, 2009.

[133] S. Deshmukh, B. Natarajan, and A. Pahwa, State estimation over a lossy network in spatially distributed cyber-physical systems, IEEE Transactions on Signal Processing, vol. 62, no. 15, pp. 3911-3923, 2014.

[134] A. Klausner, A. Tengg, and B. Rinner, Distributed multilevel data fusion for networked embedded systems, IEEE Journal of Selected Topics in Signal Processing, vol. 2, no. 4, pp. 538-55, 2008. 
[135] L. Shi and L. Xie, Optimal sensor power scheduling for state estimation of Gauss-Markov systems over a packet-dropping network, IEEE Transactions on Signal Processing, vol. 60, no. 5, pp. 2701-2705, 2012.

[136] S. Sun, Optimal linear filters for discrete-time systems with randomly delayed and lost measurements with/without time stamps, IEEE Transactions on Automatic Control, vol. 58, no. 6, pp. 1551-1556, 2013.

[137] P. Zhang, W. Qi, and Z. Deng, Hierarchical fusion robust Kalman filter for clustering sensor network time-varying systems with uncertain noise variances, International Journal of Adaptive Control and Signal Processing, vol. 29, no. 1, pp. 99-122, 2015.

[138] J. Wagner, F. Lingenfelser, E. Andre, and J. Kim, Exploring fusion methods for multimodal emotion recognition with missing data, IEEE Transactions on Affective Computing, vol. 2, no. 4, pp. 206-218, 2011.

[139] Q. Liu, X. Wang, and S. V. Nageswara, Fusion of state estimates over long-haul sensor networks with random loss and delay, IEEE-ACM Transactions on Networking, vol. 23, no. 2, pp. 644-656, 2015.

[140] A. J. Terry, M. Zaman, and J. Illingworth, Sensor fusion by a novel algorithm for time delay estimation, Digital Signal Processing, vol. 22, no. 3, pp. 439-452, 2012.

[141] M. A. K. Jaradat and M. F. Abdel-Hafez, Enhanced, delay dependent, intelligent fusion for INS/GPS navigation system, IEEE Sensors Journal, vol. 14, no. 5, pp. 1545-1554, 2014.

[142] H. Dong, Z. Wang, J. Lam, and H. Gao, Distributed filtering in sensor networks with randomly occurring saturations and successive packet dropouts, International Journal of Robust and Nonlinear Control, vol. 24, no. 12, pp. 1743-1759, 2014.

[143] D. Zhang, L. Yu, and W. Zhang, Energy efficient distributed filtering for a class of nonlinear systems in sensor networks, IEEE Sensors Journal, vol. 15, no. 5, pp. 3026-3036, 2015.

[144] Q. Ren, Y. Zhu, X. Shen, and E. Song, Optimal sensor rules and unified fusion rules for multisensor multi-hypothesis network decision systems with channel errors, Automatica, vol. 45, no. 7, pp. 1694-1702, 2009.

[145] J. Feng, Z. Wang, and M. Zeng, Optimal robust non-fragile Kalman-type recursive filtering with finite-step autocorrelated noises and multiple packet dropouts, Aerospace Science and Technology, vol. 15, no. 6, pp. 486-494, 2011.

[146] F. Li, J. Zhou, and D. Wu, Optimal filtering for systems with finite-step autocorrelated noises and multiple packet dropouts, Aerospace Science and Technology, vol. 24, no. 1, pp. 255-263, 2013.

[147] Y. Liu, X. He, Z. Wang, and D. Zhou, Optimal filtering for networked systems with stochastic sensor gain degradation, Automatica, vol. 50, no. 5, pp. 1521-1525, 2014.

[148] D. Ding, Z. Wang, B. Shen, and H. Dong, Envelope-constrained $H_{\infty}$ filtering with fading measurements and randomly occurring nonlinearities: the finite horizon case, Automatica, vol. 55, pp. 37-45, 2015.

[149] Y. Liu, Z. Wang, J. Liang, and X. Liu, Synchronization and state estimation for discrete-time complex networks with distributed delays, IEEE Transactions on Systems Man and Cybernetics Part B-Cybernetics, vol. 38, no. 5, pp. 1314-1325, 2008.

[150] J. Liu, J. Cao, Z. Wu, and Q. Qi, State estimation for complex systems with randomly occurring nonlinearities and randomly missing measurements, International Journal of Systems science, vol. 45, no. 7, pp. 1364-1374, 2014.

[151] J. Liang, Z. Wang, and X. Liu, State estimation for two-dimensional complex networks with randomly occurring nonlinearities and randomly varying sensor delays, International Journal of Robust and Nonlinear Control, vol. 24, no. 1, pp. 18-38, 2014.

[152] B. Shen, Z. Wang, and X. Liu, Bounded $H_{\infty}$ synchronization and state estimation for discrete time-varying stochastic complex networks over a finite-horizon, IEEE Transactions on Neural Networks, vol. 22, no. 1, pp. 145-157, 2011.

[153] J. Hu, Z. Wang, S. Liu, and H. Gao, A variance-constrained approach to recursive state estimation for time-varying complex networks with missing measurements, Automatica, vol. 64, pp. 155-162, 2016.

[154] B. Shen, Z. Wang, and X. Liu, A stochastic sampled-data approach to distributed $H_{\infty}$ filtering in sensor networks, IEEE Transactions on Circuits and Systems-I: Regular Papers, vol. 58, no. 9, pp. 2237-2246, 2011.

[155] J. Liang, Z. Wang, and X. Liu, Distributed state estimation for discrete-time sensor networks with randomly varying nonlinearities and missing measurements, IEEE Transactions on Neural Networks, vol. 22, no. 3, pp. 486-496, 2011.

[156] B. Shen, Z. Wang, and Y. S. Hung, Distributed consensus $H_{\infty}$ filtering in sensor networks with multiple missing measurements: the finite-horizon case, Automatica, vol. 46, no. 10, pp. 1682-1688, 2010.

[157] Q. Liu, Z. Wang, X. He, and D. Zhou, Event-based recursive distributed filtering over wireless sensor networks, IEEE Transactions on Automatic Control, vol. 60, no. 9, pp. 2470-2475, 2015.

[158] J. Ma and S. Sun, Centralized fusion estimators for multisensor systems with random sensor delays, multiple packet dropouts and uncertain observations, IEEE Sensors Journal, vol. 13, no. 4, pp. 1228-1235, 2013.

[159] J. Ma and S. Sun, Information fusion estimators for systems with multiple sensors of different packet dropout rates, Information Fusion, vol. 12, no. 3, pp. 213-222, 2011. 
[160] C. Pan and S. Sun, Fusion predictors for multisensor stochastic uncertain systems with missing measurements and unknown measurement disturbances, IEEE Sensors Journal, vol. 15, no. 8, pp. 4346-4354, 2015.

[161] R. Caballero-Águila, I. García-Garrido, and J. Linares-Pérez, Information fusion algorithms for state estimation in multi-sensor systems with correlated missing measurements, Applied Mathematics and Computation, vol. 226, pp. 548-563, 2014.

[162] N. Li, S. Sun, and J. Ma, Multi-sensor distributed fusion filtering for networked systems with different delay and loss rates, Digital Signal Processing, vol. 34, pp. 29-38, 2014.

[163] J. Feng, Z. Wang, and M. Zeng, Distributed weighted robust Kalman filter fusion for uncertain systems with autocorrelated and cross-correlated noises, Information Fusion, vol. 14, no. 1, pp. 78-86, 2013.

[164] J. Sun, C. Zhang, and J. Gu, Decentralized optimal fusion filtering for multi-sensor multi-delay singular systems, Circuits Systems and Signal Processing, vol. 31, no. 1, pp. 163-176, 2012.

[165] Y. Xia, J. Shang, J. Chen, and G. Liu, Networked data fusion with packet losses and variable delays, IEEE Transactions on Systems Man and Cybernetics Part B-Cybernetics, vol. 39, no. 5, pp. 1107-1120, 2009.

[166] E. Song, J. Xu, and Y. Zhu, Optimal distributed Kalman filtering fusion with singular covariances of filtering errors and measurement noises, IEEE Transactions on Automatic Control, vol. 59, no. 5, pp. 1271-1282, 2014.

[167] M. J. García-Ligero, A. Hermoso-Carazo, and J. Linares-Pérez, Distributed and centralized fusion estimation from multiple sensors with Markovian delays, Applied Mathematics and Computation, vol. 219, no. 6, pp. 2932-2948, 2012.

[168] M. J. García-Ligero, A. Hermoso-Carazo, and J. Linares-Pérez, Distributed fusion estimation in networked systems with uncertain observations and Markovian random delays, Signal Processing, vol. 106, pp. 114-122, 2015.

[169] B. Chen, L. Yu, W. Zhang, and A. Liu, Robust information fusion estimator for multiple delay-tolerant sensors with different failure rates, IEEE Transactions on Circuits and Systems-I: Regular Papers, vol. 60, no. 2, pp. 401-414, 2013.

[170] M. Liu, D. Qi, S. Zhang, M. Qiu, and S. Zheng, Optimal $H_{\infty}$ fusion filters for a class of discrete-time intelligent systems with time delays and missing measurement, Neurocomputing, vol. 74, no. 18, pp. 3741-3752, 2011. 